\title{
Features of compressed rods calculations with account of initial imperfections and creep effects
}

\author{
Viacheslav Chepurnenko ${ }^{1, *}$, Batyr Yazyev $^{1}$, and Ludmila Dubovitskaya ${ }^{2}$ \\ ${ }^{1}$ Don State Technical University, Faculty of Civil Engineering, Gagarin 1 Sq., 344000, Rostov-on-Don, \\ Russia \\ ${ }^{2}$ Russian State Tourism and Service University, 99 Glavnaya street, Moscow, 141221, Russia
}

\begin{abstract}
The article presents solutions to the problem of rod buckling, taking into account creep effects. Trigonometric series, the finite difference method in combination with the programming language MATLAB, as well as the finite element method in the ANSYS software package were used in the solutions. The behavior of the rods is researched for two types of relations between strain and stress during creep, with strains in an explicit and implicit form. When solving, the criterion of initial imperfections with their different values is used, as well as the tangential-modular theory. The results obtained for the two creep models are compared. The conclusion is made about the accuracy of the results of calculations in ANSYS with the presence of a combination of geometric and physical nonlinearity for various creep models.
\end{abstract}

\section{Introduction}

Buckling analysis of compressed rods considering creep effects has been touched on many articles, including [1-20]. The articles [1-3] outline the solution to this problem using the finite difference method. In the works [4-5] the energy method in the form of RitzTymoshenko is applied. The finite element method in the form of the Bubnov-Galerkin method [6-9] can also be used to calculate compressed rods for creep. Problems of dynamic stability in creep are discussed in the papers [10-16]. In the articles [17-19], the apparatus of the theory of probability is connected to calculate viscoelastic rods. None of these publications compare the results obtained by different methods. Also of great interest is the comparison of solutions using well-known numerical and numerical-analytical methods with the results obtained in modern FEM complexes (ANSYS, Abaqus, etc.). The purpose of the study is to assess the reliability of the results obtained using FEM complexes.

\footnotetext{
* Corresponding author: chepurnenkoviacheslav@gmail.com
} 


\section{Methods}

We consider a rod joint-connected to the ground at the ends with an axis initially curved according to the equation $v_{0}=f_{0} \sin \frac{\pi x}{l}, 0 \leq x \leq l$, in the plane of least rigidity and loaded with a force $P$ (Fig. 1.).

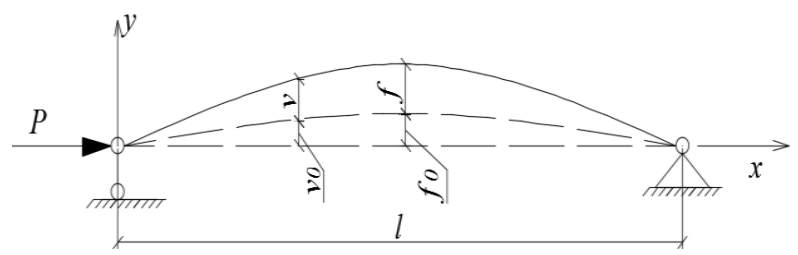

Fig. 1. Loading model.

The solution of the problem is reduced to the following differential equation (1) regarding the magnitude of the deflection $v[6]$ :

$$
\frac{\partial^{2} v}{\partial x^{2}}+\frac{P v}{E I}=-\frac{1}{I} \int_{A} \varepsilon^{*} y d A-\frac{P v_{0}}{E I} .
$$

Here $\varepsilon^{*}-$ creep strain, $A$ - cross section area. It is assumed that the stresses in the rod do not exceed the elastic limit until the moment of buckling, and also that the EulerBernoulli beam theory simplification is fulfilled. The study will be conducted on the example of a dural rod with a length of $l=21.4 \mathrm{~cm}, f_{0}=0.01 \mathrm{~cm}$, of rectangular cross section $(b=2 \mathrm{~cm}, h=0.8 \mathrm{~cm})$, which is loaded by the central compressive force $P=$ $=500 \mathrm{~kg}$. At $T=300{ }^{\circ} \mathrm{C} E=4 \cdot 10^{5} \mathrm{~kg} / \mathrm{cm}^{2}$, the creep law is represented as [20] :

$$
\varepsilon^{*}=5 \cdot 10^{-12} \sigma^{3} t
$$

where hours are time units, $\sigma$ is measured in $\mathrm{kg} / \mathrm{cm}^{2}$.

The solution of the differential equation (1) is produced by the finite difference method using the program developed by the authors in the MATLAB package. The differential equation is transformed into a system of linear equations that has the form:

$$
[A] \cdot\{V\}=\{B\}
$$

where

$$
[A]=\left(\begin{array}{ccccc}
\frac{P}{E I}-\frac{2}{\Delta x^{2}} & \frac{1}{\Delta x^{2}} & 0 & \cdots & 0 \\
\frac{1}{\Delta x^{2}} & \frac{P}{E I}-\frac{2}{\Delta x^{2}} & \frac{1}{\Delta x^{2}} & \cdots & 0 \\
\vdots & \vdots & \vdots & \ddots & \vdots \\
0 & \cdots & \cdots & \frac{1}{\Delta x^{2}} & \frac{P}{E I}-\frac{2}{\Delta x^{2}}
\end{array}\right),
$$


$[A]$ is $(n-1) \times(n-1)$ matrix, $n$ is the number of segments into which the length of the rod $l$

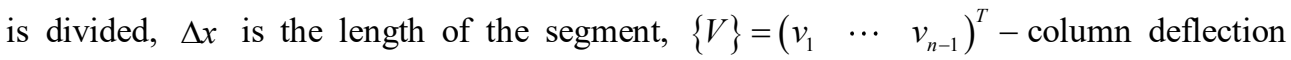
matrix, $\{B\}=\left(\begin{array}{lll}b_{1} & \cdots & b_{n-1}\end{array}\right)^{T}$, elements $b_{i}$ are equal to:

$$
b_{i}=-\frac{1}{I} \int_{-\frac{h}{2}}^{+\frac{h}{2}} \varepsilon^{*}(i \cdot \Delta x, y, t) y b d y-\frac{P v_{0}(i \cdot \Delta x)}{E I} .
$$

Calculations are being produced step by step with time intervals $\Delta t$. At each moment, we calculate stresses using the formula (6):

$$
\sigma=\frac{E}{A} \int_{A} \varepsilon^{*} d A-\frac{P}{A}-E y \frac{\partial^{2} v}{\partial x^{2}}-E \varepsilon^{*} .
$$

The integrals are being found numerically by the trapezoidal rule using the built-in function «trapz ()». Prof. Volmir has realized solution of this problem using trigonometric series in the monograph [20]. We precise this solution by increasing the number of time steps, as well as the number of nodes along the cross section height. We perform calculations with MATLAB.

The differential equation for the deflection increments over the time interval $\Delta t$ is as follows:

$$
\frac{\partial^{2} \Delta v}{\partial x^{2}}+\frac{P}{E I} \Delta v=\frac{P}{E I}\left(-\frac{E}{P} \int_{A} \Delta \varepsilon^{*} y d A\right) .
$$

The expression in parentheses is decomposed in the series below.:

$$
-\frac{E}{P} \int_{A} \Delta \varepsilon^{*} y d A=\sum_{i=1}^{S} \Delta a_{i} \sin \frac{i \pi x}{l} .
$$

The solution of equation (5) is also decomposed in the series:

$$
\Delta v=\sum_{i=1}^{S} \Delta b_{i} \sin \frac{i \pi x}{l}
$$

For convenience, we take $S$ equal to the number of nodes along the length of the rod with nonzero deflections and coordinates $x_{i}$. Then we will find $\Delta a_{i}$ based on the solution of the system of equations for a certain point in time. In matrix form, the system is written as:

$$
[K]\{R\}=\{C\}
$$

where: 


$$
[K]=\left[\begin{array}{cccc}
\sin \frac{\pi x_{1}}{l} & \sin \frac{2 \pi x_{1}}{l} & \cdots & \sin \frac{s \pi x_{1}}{l} \\
\sin \frac{\pi x_{2}}{l} & \sin \frac{2 \pi x_{2}}{l} & \cdots & \sin \frac{s \pi x_{2}}{l} \\
\vdots & \vdots & \ddots & \vdots \\
\sin \frac{\pi x_{s}}{l} & \sin \frac{2 \pi x_{s}}{l} & \cdots & \sin \frac{s \pi x_{s}}{l}
\end{array}\right]
$$

$\{R\}=\left(\begin{array}{lll}\Delta a_{1} & \cdots & \Delta a_{s}\end{array}\right)^{T},\{C\}=\left(\begin{array}{lll}c_{1} & \cdots & c_{s}\end{array}\right)^{T}$, matrix elements are equal to:

$$
c_{i}=-\frac{E}{P} \int_{A} \Delta \varepsilon^{*}\left(x_{i}, y, t\right) y d A .
$$

In the above expression for $c_{i}$ :

$$
\Delta \varepsilon^{*}\left(x_{i}, y, t+\Delta t\right)=C_{c r}\left(\sigma\left(x_{i}, y, t\right)\right)^{3} \Delta t
$$

$C_{c r}$ is the creep law constant in (2). The integral is calculated analogously numerically for each of the nodes with $x_{i}$ coordinates. The increments of the coefficients will be equal to:

$$
\Delta b_{i}=-\frac{\Delta a_{i}}{i^{2} \pi^{2} E I /\left(P l^{2}\right)-1},
$$

stress increments are equal to:

$$
\Delta \sigma=E \Delta \varepsilon=E\left(\Delta \varepsilon_{0}-\Delta \chi y-\Delta \varepsilon^{*}\right),
$$

where:

$$
\Delta \varepsilon_{0}=\frac{1}{A} \int_{A} \Delta \varepsilon^{*} d A
$$

is the increment of the axial deformation of the neutral line of the rod,

$$
\Delta \chi=-\sum_{i=1}^{S} \Delta b_{i} \frac{i^{2} \pi^{2}}{l^{2}} \sin \frac{i \pi x}{l}
$$

is curvature increment of the neutral line.

\section{Results and discussions}

The results of calculation by finite differences method are presented in Fig. 2. On the graphs "nt" is the number of steps in time taken in the calculations. As you can see, with an increase in the number of time intervals, convergence is observed. At the time moment $t$ $>0.6 \mathrm{~h}$, a sharp growth of the deflection arrow occurs, the bearing capacity of the rod is exhausted. 
The resulting solution by modified prof. Volmir's method is presented in Fig. 3. The numbers of steps in time is equal to 1000 in the calculations. As can be seen on the graph, the critical time comes in $0.6 \ldots 0.65 \mathrm{~h}$ after loading. These values almost coincide with the results found using the finite difference method.

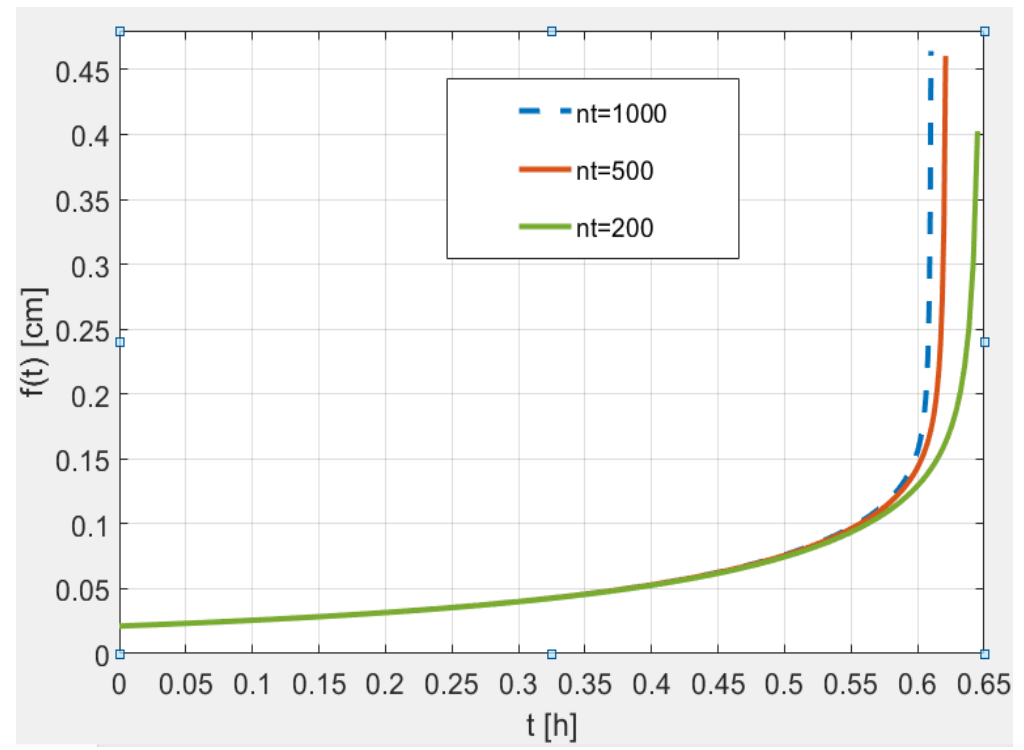

Fig. 2. Finite difference method results.

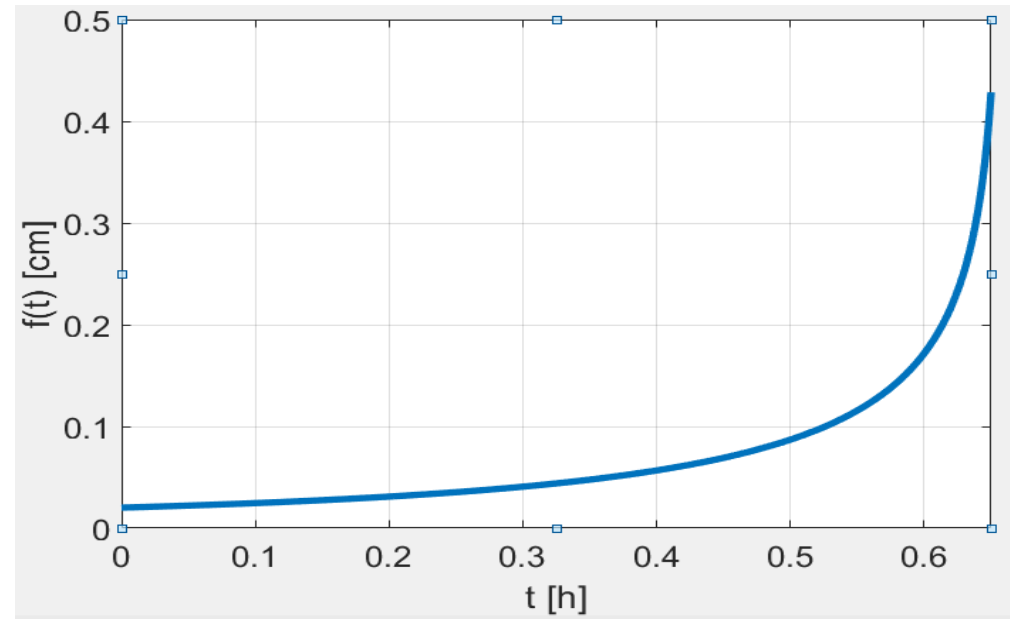

Fig. 3. Result using prof. Volmir's method.

We also use the ANSYS software package for solving the previously described problem by the finite element method. First, we use the beam elements. Material behavior is setting up using the Modified Time Hardening [21] creep model:

$$
\varepsilon_{c r}=\frac{C_{1} \sigma^{C_{2}} t^{C_{3}+1} e^{-C_{4} / T}}{C_{3}+1}
$$


Coefficients are equal to $C_{1}=1.389 \cdot 10^{-30} \mathrm{~m}^{6} /\left(\mathrm{kg}^{3} \cdot \mathrm{s}\right), C_{2}=3, C_{3}=C_{4}=0$ for our problem. Maximum time step was specified as 7 seconds.

As can be seen on the Fig. 4, the critical time, based on the obtained results, $t>7000 \mathrm{~s}$ $\approx 1.95 \mathrm{~h}$, while the value of the critical time when solving problem with the previously described methods is within $0.6 \ldots 0.7 \mathrm{~h}$, which is about 3 times less than that obtained in ANSYS. Next we simulate the behavior of the rod using flat elements PLANE183 (Fig. 5).

In order to avoid errors produced by the software package the creep effects were disregarded in the vicinity of the point of application of force and the point of fixation due to the singularities there. The dependence of the deflection arrow on time is depicted in Fig. 6.

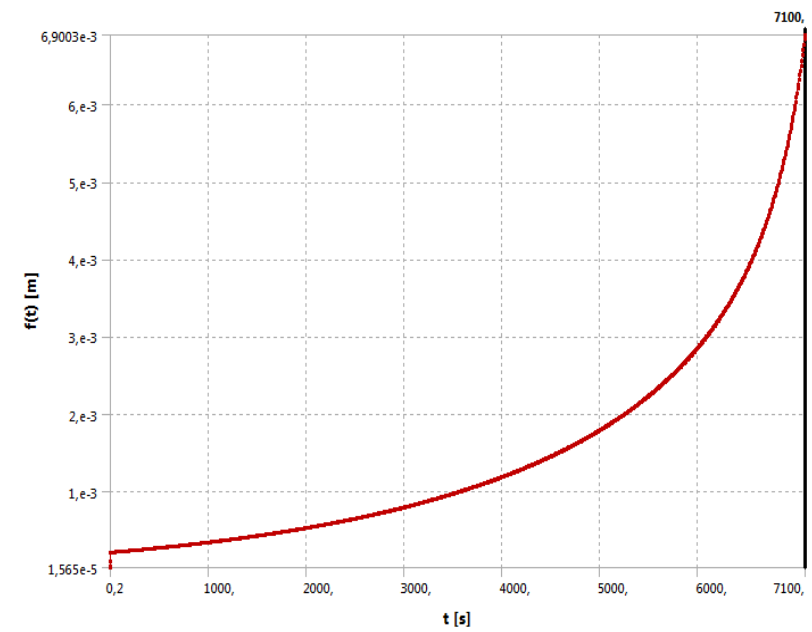

Fig. 4. BEAM188 elements result

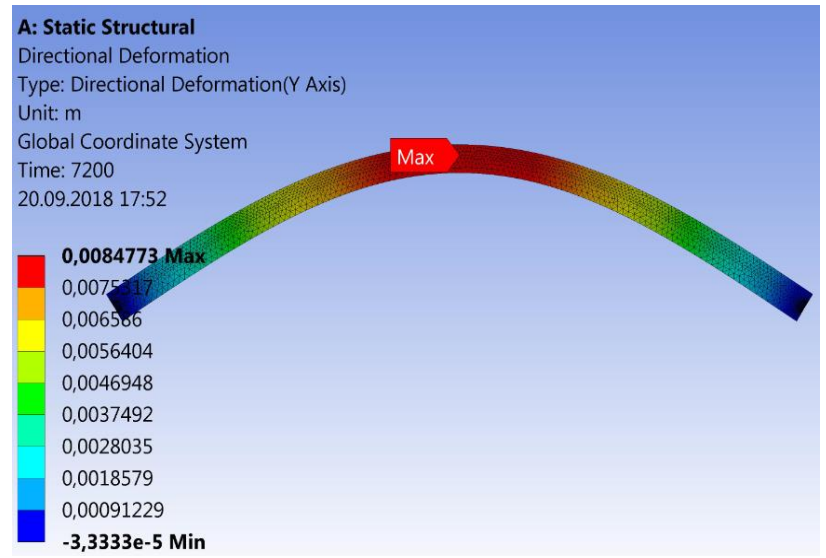

Fig. 5. Deformed model $(\times 5)$ consisting of Plane 183 elements 


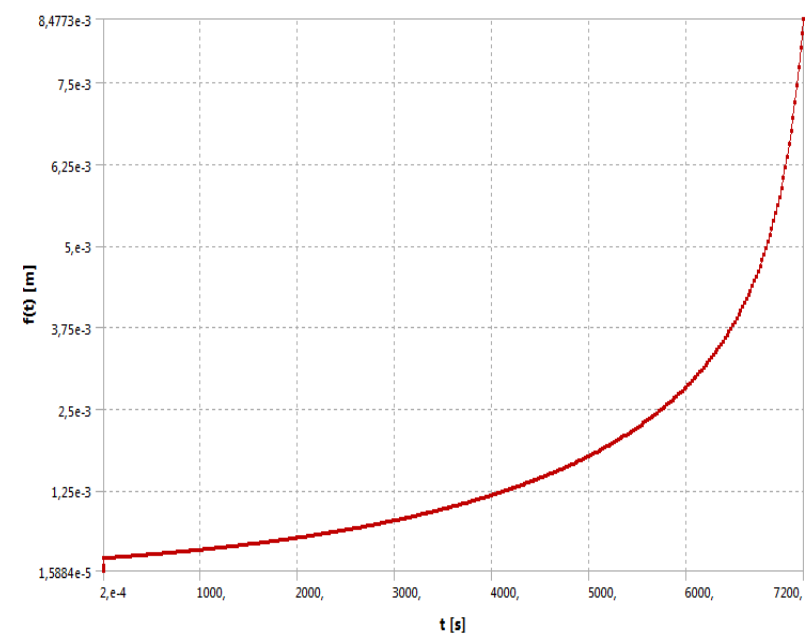

Fig. 6. PLANE183 elements result.

In this case, the loss of bearing capacity occurs in the period $t=7100 \ldots 7200 \mathrm{~s}$. These values are also significantly superior to those obtained earlier by the finite difference method.

Next, we take a rod with a shape closer to the ideal one, $f_{0}=0.001 \mathrm{~cm}$, which is 10 times smaller than the value previously used. The critical time takes on a value in the vicinity of $t=3150 \mathrm{~s}$, which amounts to $0.88 \mathrm{~h}$ (Fig. 7).

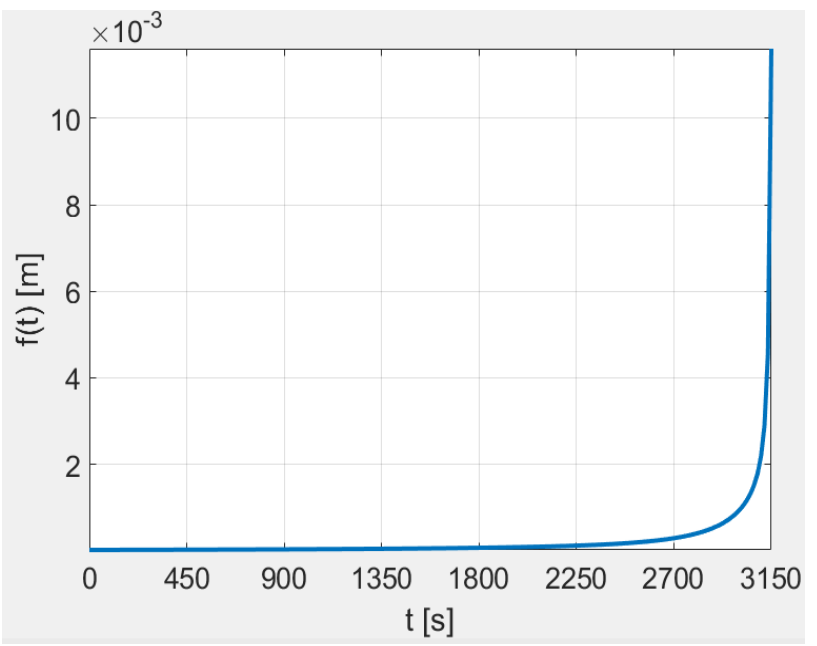

Fig. 7. Finite difference method results with small initial deflections.

We use the tangential-modular theory for determining the critical time and compare the obtained result and the calculation with the initial imperfections of the straight axis of the rod close to zero. With respect to the modular theory, the critical time will be equal to:

$$
t_{*}=\frac{1}{n} \frac{1-\bar{\sigma}_{*}}{E k \bar{\sigma}_{*}^{n} \bar{\sigma}_{e}^{n-1}}=0.83 \mathrm{~h}
$$


Here $\bar{\sigma}_{*}=\bar{\sigma} / \bar{\sigma}_{e}=P /\left(A \bar{\sigma}_{e}\right)$, where $\bar{\sigma}_{e}=\pi^{2} E i^{2} / l^{2}-$ Euler stress, $i$ is the minimum cross section radius of inertia. The values obtained by these two methods almost coincide.

We will compare further the results obtained in ANSYS and MATLAB without taking into account the geometric nonlinearity for the initially specified data. Modifying the differential equation (1) accordingly, we obtain the following expression:

$$
\frac{\partial^{2} v}{\partial x^{2}}=-\frac{1}{I} \int_{A} \varepsilon^{*} y d A-\frac{P v_{0}}{E I} .
$$

In ANSYS, in the analysis settings, we disable the "Large deflections" option, for modeling we also use BEAM188 elements. The results are shown in Figure 8.

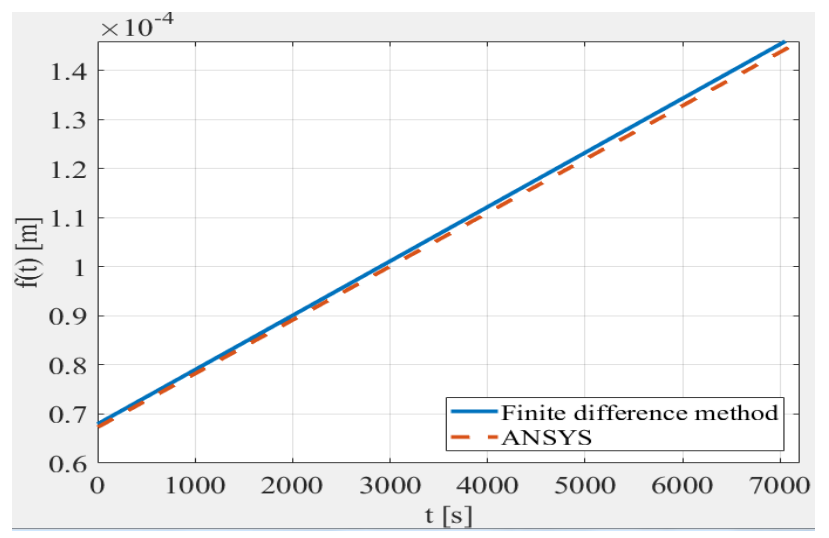

Fig. 8. Results without nonlinear effects.

As we can see, the graphs obtained in the calculation by the finite difference method and the finite element method in the ANSYS software package overlap each other with a small error. At time $\mathrm{t}=7200 \mathrm{~s}$, the difference between the deflection arrow values obtained in two ways is $\Delta=1.6464 \cdot 10^{-6} \mathrm{~m}, \delta=1.1 \%$.

Note that the feature of the previously considered model was that there were explicit creep strains and stresses in the creep law. Next we perform the calculation for the rod made of alloy AK4-1T. The load on the rod is equal to $P=8000 \mathrm{~N}$. In accordance with [22], the law of material creep is adopted in the form:

$$
\frac{\partial \varepsilon^{*}}{\partial t}=B \sigma|\sigma|^{n-1}
$$

where $B$ and $n$ are material constants.

At the temperature $T=200^{\circ} \mathrm{C}$, alloy has the following properties: $E=0.6 \cdot 10^{5} \mathrm{MPa}$; constants of the creep law take the values: $n=7, B=3.7152 \cdot 10^{-62} \mathrm{~Pa}^{-7} \cdot \mathrm{s}^{-1}$. This law corresponds to the Strain Hardening model in ANSYS: $\dot{\varepsilon}_{c r}=C_{1} \sigma^{C_{2}} \varepsilon_{c r} e^{C_{3}} e^{-\frac{C_{4}}{T}}$, accordingly, the coefficients will be equal $C_{3}=C_{4}=0$. Also we perform the calculation using the finite difference method. We will do the calculations step by step. For small time increments, based on the definition of the derivative, the expression for the partial derivative takes the form: 


$$
\frac{\partial \varepsilon^{*}}{\partial t} \approx \frac{\Delta \varepsilon^{*}}{\Delta t}=B \sigma^{7}
$$

from where:

$$
\Delta \varepsilon^{*}=B \sigma^{7} \Delta t
$$

We will build the computational process as follows: we find the solution without creep, at the moment, further, using the found stresses, we calculate the creep deformation increments using formula (22). Then we obtain the creep deformations at the moment of time $t+\Delta t$, using the formula (23):

$$
\varepsilon^{*}(t+\Delta t)=\varepsilon^{*}(t)+\Delta \varepsilon(t)^{*}
$$

The results are shown on the Fig. 9.

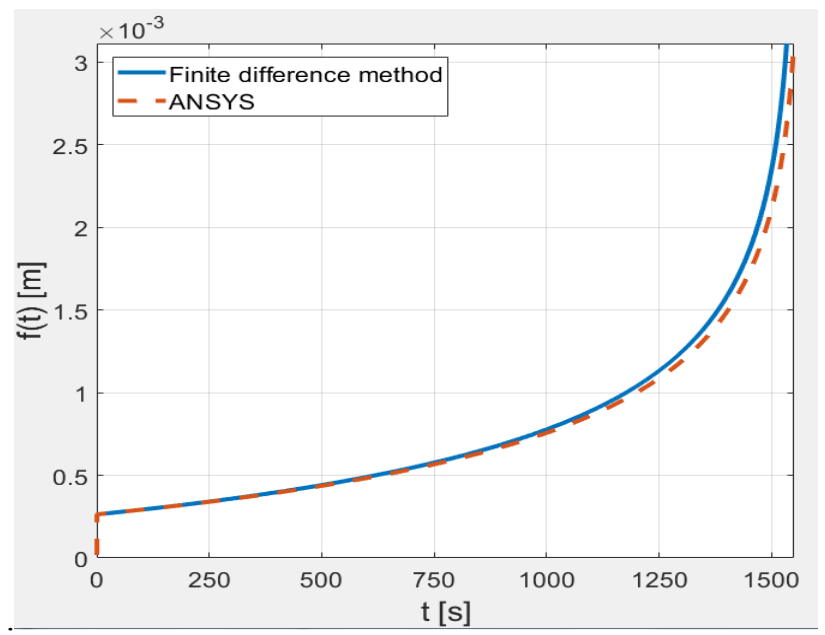

Fig. 9. Matching results obtained with finite difference method and ANSYS.

When solving by the finite difference method, a time step of $0.6 s$ was taken. The solution in ANSYS corresponds to the number of substeps equal to 400. It is easy to see that when using the creep law in differential form, the results obtained by the finite difference method using the MATLAB programming language and the finite element method in ANSYS differ insignificantly. The sharp growth of the deflection in both cases is observed after $1520 \mathrm{~s}$.

\section{Conclusions}

The presented results show that in the case of the use of the Modified Time Hardening creep law, the critical time defined in the ANSYS software package is greatly overestimated compared to solutions by other methods. At the same time, for geometrically linear problems and when specifying the creep law in differential form, the discrepancy is insignificant. This fact should be taken into account in the design of structural elements, which are characterized by high-temperature creep. 


\section{References}

1. S.V. Litvinov, E.S. Klimenko, I.I. Kulinich, S.B. Yazyeva, Int. Polym. Sci. Technol. 42, 2 (2015)

2. V.I. Andreev, N.Y. Tsybin, Procedia Eng. 111, $42-48$ (2015)

3. V.I. Andreev, E.V. Barmenkova, Procedia Eng. 91, 20-25 (2014)

4. S.B. Yazyev, M. Yu. Kozelskaya, G.P. Strelnikov, S.V. Litvinov, MATEC Web Conf. 129, 05010 (2017)

5. V.I. Andreev, B.M. Yazyev, A.S. Chepurnenko, Adv. Mater. Res. 1004-1005, 257-260 (2014)

6. B.M. Yazyev, S.B. Yazyev, A.P. Grinev, E.A. Britikova, Mater. Sci. Forum 931, $127-$ 132 (2018)

7. G.M. Danilova-Volkovskaya, A.S. Chepurnenko, A. Begak, A.A. Savchenko, IOP Conf. Ser. Earth Environ. Sci. 90 (2017)

8. A.A. Veremeenko, A.S. Chepurnenko, P.A. Shvetsov, L.A. Zorchenko, S.B. Yazyev, IOP Conf. Ser. Earth Environ. Sci. 90 (2017)

9. J. Walczak, J. Sieniawski, K.J. Bathe, Comput. Struct. 17, 783-792 (1983)

10. T.A. Aslan, A.R. Noori, B. Temel, Mech. Res. Commun. 92, 8-14 (2018)

11. Y. A. Chirkunov, J. Appl. Math. Mech. 79, 506-513 (2015)

12. N. Eratli, H. Argeso, F.F. Çalim, B. Temel, M.H. Omurtag, J. Sound Vib. 333, 36713690 (2014)

13. B.A. Khudayarov, F.Z. Turaev, Appl. Math. Model. 66, 662-679 (2019)

14. Z. Ding, L. Li, J. Kong, L. Qin, Comput. Struct. 194, 60-73 (2018)

15. Y. Liu, M. Wang, Mech. Res. Commun. 70, 17-23 (2015)

16. H. Lang, M. Arnold, Appl. Numer. Math. 62, 1411-1427 (2012)

17. V.D. Potapov, J. Appl. Math. Mech. 56, 90-95 (1992)

18. V.D. Potapov, A.Y. Marasanov, Int. J. Solids Struct. 34, 1367-1377 (1997)

19. J. Deng, W.C. Xie, M.D. Pandey, J. Sound Vib. 333, 6121-6139 (2014)

20. A.S. Volmir, Stability of deformable systems, (Moscow, Fizmatlit, 1967)

21. E. Madenci, I. Guven, The Finite Element Method and Applications in Engineering Using ANSYS (Springer International Publishing, London, 2015)

22. I.D. Klopotov, S.Y. Nizovskih, Applied mechanics and engineering physics 256, 6 (2002) 